\title{
LAS TRANSFORMACIONES TERRITORIALES Y EL DESARROLLO RURAL TERRITORIAL EN LA PROVINCIA DEL CHACO.
}

\section{TERRITORIAL TRANSFORMATIONS AND RURAL DEVELOPMENT IN THE PROVINCE OF CHACO.}

\author{
Mgter. Prof. Viviana Pértile \\ Prof. Adjunta Cátedra: Geografía Urbana y Agraria \\ Departamento de Geografía - Facultad de Humanidades -UNNE- \\ (vpertile@gmail.com). \\ (vpertile@hum.unne.edu.ar)
}

\section{RESUMEN}

La cuestión rural en la Argentina lleva consigo una discusión intensa, prolongada y sin resolver. La persistencia de la pobreza, la creciente desigualdad y el despoblamiento de amplias zonas rurales de nuestro país y de la provincia del Chaco, hablan de la poca efectividad que tuvieron las políticas públicas de desarrollo rural impulsadas desde hace varias décadas en el territorio nacional. Es evidente que en general se ha tratado de una serie de propósitos discursivos, por cuanto no fueron diseñadas ni definidas desde los ámbitos locales, prueba de ello son las transformaciones territoriales como así también los contrastes sociales y económicos que se han producido tanto en ámbitos rurales nacionales como provinciales.

En este trabajo se intenta poner de manifiesto la relación existente entre desigualdad, territorio y desarrollo, en el marco de las políticas de desarrollo rural territorial dirigidas teóricamente a beneficiar a familias de escasos recursos y vinculadas con acciones que surgen desde los actores locales. Para ello nos centramos en la región del centro y sudoeste chaqueño, donde el cultivo de algodón, principal actividad de miles de pequeños productores, entra en disputa por el territorio, como consecuencia de la alta rentabilidad que adquiere el cultivo de la soja transgénica. Ello pone en evidencia las transformaciones socio económico y territorial que se dan como consecuencia del avance de la frontera agropecuaria en el marco de las implicancias de la globalización en ámbitos locales.

\section{PALABRAS CLAVES: Territorio- Desarrollo- Rural-Políticas Públicas}

\begin{abstract}
The rural issue in Argentina entails an intense, prolonged and unsolved debate. The persistent poverty, the growing inequality and the depopulation of large rural areas in our country and in the province of Chaco, show the poor effectiveness of the rural development policies promoted for decades in the national territory. It is clear that in general it has been a series of discursive purpose, as they were neither designed nor defined from the local areas. Proof of this are the territorial transformations as well as the social and economic contrasts occurred in both national and provincial rural areas.
\end{abstract}


This paper attempts to show the relationship between inequality, territory and development, within the framework of the policies on territorial rural development, theoretically addressed to benefit poor families and linked to actions that arise from local actors. To do this we focus on the region of Central and Southwest Chaco, where the cultivation of cotton - main activity of thousands of small producers - enters into dispute over the territory, as a result of the high profitability that acquires the cultivation of GM soy. This exposes the socio-economic and territorial transformations that occur as a consequence of the advance of the agricultural frontier in the context of the implications of globalization on local ambits.

KEY WORDS: Territory-Development - Rural- Public Policies 
Revista Geográfica Digital. IGUNNE. Facultad de Humanidades. UNNE. Año 11. № 22.

Julio - Diciembre 2014. ISSN 1668-5180 Resistencia, Chaco

\section{Desarrollo y Territorio}

El desarrollo territorial rural es un concepto complejo que se utiliza permanentemente en la bibliografía sin que se proporcione una definición en profundidad del término. Ello se debe fundamentalmente a que no existe una sola definición para las palabras que forman este concepto, en cuyo caso implican ideas centrales con interpretaciones muy diversas de acuerdo al enfoque que se utiliza.

En primer lugar debemos decir que Desarrollo y Territorio son realidades ya que se trata de algo que podemos ver directa o indirectamente; son conceptos, ya que los utilizamos como tales y también son políticas por que forman parte de políticas públicas que utilizan los gobiernos; muchas veces se utiliza de distinta manera hasta en una misma propuesta política, por ejemplo describiendo una realidad de una determinada manera.

El Desarrollo es un término, es un concepto y una realidad, es un término porque lo usamos como cualquier palabra que define algo y generalmente se define tal cual aparece en el diccionario: acción y efecto de desarrollar. Cuando se lo vinculan con la economía se lo asocia con el progreso, pero no siempre el desarrollo está vinculado con lo que pensamos que es el progreso. Por lo tanto es un término de uso común pero también es un concepto que, incluido dentro de una propuesta de desarrollo rural territorial, debe estar perfectamente definido, es decir qué se entiende por desarrollo.

El desarrollo también puede estar asociado con algo que ya aconteció, por ejemplo cuando expresamos "el desarrollo del nordeste argentino", es decir lo que pasó y unido a eso se conecta al desarrollo futuro, es decir lo que se pretende para un determinado espacio, entonces es un término, es un concepto y es una definición de política. El proceso de entender y dar el sentido estricto a las palabras es importante porque las palabras, como estos conceptos están en continua evolución, van cambiando a lo largo de la historia y en este proceso es donde intervienen los diferentes intereses, incluso los de poder. De modo que si existe una definición de desarrollo, no es una definición casual, sino que es una definición que se encuentra vinculada con intereses de poder, es por ello que existe una gran variedad de definiciones de desarrollo.

Según Esteva (1996) la palabra desarrollo comienza en la historia latinoamericana en el período de posguerra, asociada a la propuesta de generar un desarrollo en América Latina; esta idea fue plasmada por el presidente de EEUU, Harry Truman cuando en su discurso de asunción presidencial en el año 1949 menciona la palabra subdesarrollo para posicionarse y expresar que los Estados Unidos de Norteamérica tiene que cuidar a ese mundo Latinoamericano que es subdesarrollado y generar desarrollo manifestándolo de la siguiente manera "Debemos emprender un nuevo programa audaz que permita que los beneficios de nuestros avances científicos y nuestro progreso industrial sirvan para la mejoría y el crecimiento de las áreas subdesarrolladas"

Desde ese momento y a partir de los años 60 comienzan a aparecer una serie de propuestas en donde el desarrollo estuvo ligado al territorio, de allí la importancia de asociar estos dos conceptos en forma conjunta. Muchos economistas sostenían que una de las cuestiones directamente relacionada con el subdesarrollo era la cuestión de la tenencia de la tierra haciendo alusión a los latifundios improductivos. Es en estas instancias cuando avanzan formas de gobiernos de perfil desarrollista en América Latina. Primero se habló de desarrollo Nacional (1), surgen así programas, planes y organismos como el Consejo Federal de Inversiones (CFI, 1959) (2) y el Consejo Nacional de Desarrollo (CONADE, 1961) (3). Al respecto Manzanal (2013) manifiesta que "esas políticas de intervención pública comenzaron a diseñarse y difundirse, como una opción necesaria para que el mundo subdesarrollado superara su condición marginal y se asemejara en su estructura a los países desarrollados e identificados, más tarde, como primer mundo".

A partir de la aplicación de esos planes y programas, se pudo ver que todos los países latinoamericanos se habían desarrollado en forma desigual, donde era posible observar un centro más desarrollado y el resto más marginal. En el caso de la Argentina el ejemplo más característico fue la pampa húmeda mucho más desarrollada que el resto del territorio nacional. Diferentes referentes políticos, económicos, sociólogos, decían que había que lograr un desarrollo regional, es

Publicado en formato digital: Mgter. Prof. Viviana Pértile. LAS TRANSFORMACIONES TERRITORIALES Y EL DESARROLLO RURAL TERRITORIAL EN LA PROVINCIA DEL CHACO. Revista Geográfica Digital. IGUNNE. Facultad de Humanidades. UNNE. Año 11. NN 22. Julio - Diciembre. 2014. ISSN 1668-5180 Resistencia, Chaco. 
entonces cuando aparece por primera vez la palabra regional asociada a desarrollo y surge así lo que se conoce como desarrollo regional. Esto recién se da en los primeros años de la década de 1960, con el surgimiento de la Alianza para el Progreso (1961), a partir de la cual se difundió masivamente la planificación nacional y regional del desarrollo. (Manzanal y Schneider, 2010) (4)

A partir de ese momento el término desarrollo ha tenido una gran cantidad de calificativos como: Desarrollo Local, Desarrollo Territorial, Desarrollo Ambiental, Desarrollo Sustentable, Desarrollo Rural Territorial. Esto se debió fundamentalmente a que América Latina desde los años 60 ', tal cual lo decíamos anteriormente, busca políticas de desarrollo tendientes a superar las diferencias, sin embargo se advierte que esas desigualdades existentes continúan o se han profundizado(5). El desarrollo estuvo siempre, de distintas formas, ligado al territorio; empieza como desarrollo regional, luego se hace territorial, después se hace rural. Lo que no se puede negar, al decir de Manzanal (2013) es que detrás de todas las concepciones e ideas hay expresiones de poder que conducen a generar confusiones al momento de elaborar proyectos y programas tendientes a producir algún tipo de desarrollo.

\section{Desarrollo y desigualdades}

Si detrás de la idea de desarrollo se encuentran las relaciones de poder o disputas de poder, entonces cabe preguntarnos si estos conceptos son opuestos o complementarios. Generalmente se tiende a pensar que son conceptos en oposición, es decir, si se genera más desarrollo o aumenta el desarrollo, disminuyen las desigualdades, que es por lo que se lucha a través de los planes de gobiernos, las organizaciones, es decir se trabaja para generar más desarrollo y menor desigualdad.

También se puede decir que se trata de dos conceptos complementarios, es decir a mayor desarrollo mayor desigualdad. Si el desarrollo es una expresión de relaciones de poder y si detrás de ese concepto de desarrollo hay una disputa de poder, entonces hay algo engañoso, porque en ningún plan o programa de desarrollo se dice que el objetivo es producir más desarrollo para lograr mayor concentración; pero la realidad, demostrada a través de informes -Cepal 2012 op cit- nos indica que cada vez hay mayores desigualdades. De modo que desde esta mirada, podemos decir que detrás de ese concepto de desarrollo hay otra realidad, otros intereses en juego, tendientes a un desarrollo concentrado y a favor de la extranjerización y en ese sentido el desarrollo es complementario, conducente, ya que conduce a mayor desigualdad.

El territorio del Centro y Sudoeste Chaqueño constituye un real ejemplo de lo dicho anteriormente; se trata del área dedicada al cultivo de la soja transgénica en la provincia del Chaco, cuyo crecimiento y expansión pudo darse gracias a inversiones y aportes de capitales extra provinciales y extranjeros, accediendo a las superficies desforestadas y cultivables a través de compras o arrendamiento, a muy bajo precio y desplazando al pequeño productor, imposibilitado de acceder a modernas tecnologías que acompañan al cultivo.

Esta expansión del cultivo benefició no solo al sector privado, dado su alta rentabilidad, sino también a los gobiernos gracias a las importantes recaudaciones producto de los impuestos y retenciones a las exportaciones.

Pertile y Torre Geraldi (2009) sostienen "Los grandes productores.... que en la actualidad representan el $80 \%$ de los productores de la región, adquirieron tierras en el occidente chaqueño confundiéndose las fronteras agropecuarias con la provincia de Santiago del Estero. Arrendaron y adquirieron los campos con superficies superiores a 500 hectáreas.... esta ola de inversiones que vive el campo también impulsa un proceso de concentración en la tenencia de la tierra... [...] la concentración va a seguir por un problema de escala, si se trabaja con pequeños campos, no solo no se obtienen ganancias sino que se corre el riesgo de perder plata. Una familia para vivir bien necesita, por lo menos 700 hectáreas y una empresa 5000... [...]. Las jurisdicciones departamentales han presentado el incremento de explotaciones agropecuarias que superan las 1000 hectáreas generado por la adquisición y concentración de tierras por parte de los grandes productores. Este proceso de atomización ha provocado que el $60,9 \%$ de los departamentos de la provincia del Chaco hayan perdido un número importante de pequeñas y medianas explotaciones pertenecientes a 
aquellos productores familiares que no han logrado sostener en el tiempo una estabilidad económicaproductiva.

Es indudable que esta situación profundiza las desigualdades sociales, aumentando las condiciones críticas de vida de los pequeños productores (6) y generando por otra parte mayores ganancias para las empresas o grandes propietarios.

De modo que, el ámbito rural de la provincia del Chaco, presenta algunas características del "nuevo mundo" de Manuel Castells (1998), visualizada en la convivencia de grandes sectores excluidos y desconectados del sistema, al lado de aquellos que se hallan vinculados a la sociedad red y al capitalismo informacional; en particular y como más representativo se pueden mencionar a los grandes emprendimientos agrícolas con funcionamiento autónomo respecto a la economía zonal y regional. En este marco son mucho más los trabajadores genéricos reemplazables, discontinuos y con bajo valor de las remuneraciones, que los auto programables y con capacidad de adaptarse y reconvertirse para sobrevivir a este nuevo contexto socioeconómico y político.

En este sentido, Manzanal (2011) sostiene que "se debe considerar el desarrollo (la expansión y reproducción del sistema capitalista) como un proceso esencialmente contradictorio y conflictivo, que está determinado por la respectiva estructura de relaciones de poder y dominación, objetivadas a través de normas, leyes y reglamentaciones, que conforman el aparato institucional hegemónico de cierto momento y lugar"

En virtud de lo expuesto se debería pensar en otras definiciones de Desarrollo, sobre todo si se considera que el desarrollo es un proceso conflictivo y contradictorio donde juegan sectores sociales que se encuentran enfrentados o en oposición y con diferentes intereses en juego.

En la mayoría de los proyectos de Desarrollo Territorial aparecen un conjunto de indicaciones sobre qué es lo que hay hacer o qué se debe buscar; muchos de ellos sostienen que hay que buscar territorios cuyos actores promuevan acciones a partir del consenso. Una de las condiciones para que pueda darse el Desarrollo Rural Territorial es la existencia de territorios con posibilidades de ser productivos, innovativos y competitivos.

\section{El Territorio y su vinculación ¿con DESARROLLO? el espacio y la región.}

Espacio, región y territorio suelen utilizarse indistintamente, aunque en determinados momentos se usaron unos más que otros; inicialmente se utilizó la denominación de espacio, después región y actualmente territorio; son conceptos que están en continuo en movimiento. El término territorio es amplio, diverso y ha complejizado su significado en el conjunto de las ciencias sociales. En geografía es muy utilizado y puede entenderse a nivel político e implica la delimitación en la cual existe una población asentada y que depende de una autoridad competente. Otra concepción desde la Geografía es la que está relacionada con las variaciones del paisaje en relación al relieve, al clima, a la ecología, entre otras.

Sin lugar a dudas el término territorio va más allá de lo que entendemos como la soberanía del Estado, es decir la circunscripción política administrativa y por ende trasciende la connotación geográfica, de localización, física, social, cultural, económica y política. En él todo sucede, es decir que estamos ante la presencia de un espacio donde las relaciones de todo tipo determinan su configuración o estructura. En este sentido Fernandes (2005) sostiene que es una consecuencia de relaciones sociales.

En la mayoría de los documentos o bibliografía cuando se refieren al territorio, toman como punto de partida al espacio físico, pero en realidad territorio significa algo por lo cual el Hombre está allí, es decir tiene algún tipo de interés en él y disputa esos intereses, o como sostiene Manzanal y otros (2011) "Ios territorios son ámbitos de disputa, de ejercicio de poder, donde la exclusión de los sectores dominados es una realidad presente históricamente".

Marcelo Souza (1995) dice que el territorio puede ser interpretado, fundamentalmente, como un espacio definido y delimitado por y a partir de relaciones de poder.

Para Schejtman y Berdegué (2004), promotores del desarrollo territorial rural, el territorio se constituye en un elemento de una trilogía que resume la diversidad social, económica y política del proceso de desarrollo tanto a nivel micro como macro, es decir pasando desde una escala local,

Publicado en formato digital: Mgter. Prof. Viviana Pértile. LAS TRANSFORMACIONES TERRITORIALES Y EL DESARROLLO RURAL TERRITORIAL EN LA PROVINCIA DEL CHACO. Revista Geográfica Digital. IGUNNE. Facultad de Humanidades. UNNE. Año 11. No 22. Julio - Diciembre. 2014. ISSN 1668-5180 Resistencia, Chaco. 
regional, nacional y mundial; como así también el espacio que sus agentes reconocen como necesario para contener y delimitar las relaciones que establecen entre ellos en el interior, y entre todos y el "mundo externo", en función de los proyectos u objetivos de desarrollo que se proponen emprender. Dicho en otras palabras el territorio en cada proceso de desarrollo rural es una construcción social, y no un espacio.

"objetivamente existente" y delimitable mediante un puro ejercicio técnico ex ante, en virtud de una u otra variable o conjunto de variables físicas o económicas. La definición operacional de territorio es puramente instrumental, es decir, funcional a los objetivos y alcances del proyecto que se proponen los agentes de los procesos de desarrollo territorial rural.

Por su parte Milton Santos (1986) considera al territorio como producto histórico del trabajo humano, que deriva en la construcción de un dominio o de una delimitación de lo vivido espacialmente, donde el territorio asume múltiples formas y determinaciones: económica, administrativa, bélica, cultural y jurídica. El territorio es un área demarcada donde individuos ejercen su poder. A ello podríamos agregar que el territorio es un espacio que ha sido puesto en valorización para el capital, entonces en la medida que está valorizado, que ha sido descubierto, desde el momento en que el hombre interviene allí, es considerado una Construcción social, es producto de la acción del hombre.

Maryvonne Le Berre (1992), manifiesta que el territorio puede ser definido como la porción de la superficie terrestre, apropiada por un grupo social para asegurar su reproducción y la satisfacción de sus necesidades vitales. Todo grupo ordena y administra esa extensión de tierra que posee [...] el resultado es la producción de un territorio dotado de una cierta estabilidad en el tiempo. [...] Comprender un territorio significa primero poner en evidencia las interacciones entre un grupo social y su territorio. Esta idea de interacción podemos completarla con Bozzano, (2009), en una denominada definición provisoria de Territorio, "Es un lugar de variada escala -micro, meso, macro- donde actores - públicos, privados, ciudadanos- ponen en marcha procesos complejos de interacción complementaria, contradictoria, conflictiva, cooperativa- entre sistemas de acciones y sistemas de objetos, constituidos éstos por un sinnúmero de técnicas -híbridos naturales y artificiales- e identificables según instancias de un proceso de organización territorial en particulares acontecimientos -en tiempo-espacio- y con diversos grados de inserción en la relación local-mesoglobal. El territorio se redefine siempre"; más adelante Bozzano (op cit) dice que el territorio es una relación genérica entre sociedad y naturaleza, pudiéndose aludir tanto a procesos de alcance más general con actores de compleja identificación, como a acontecimientos más particulares donde los actores tendrán necesariamente mayor presencia.

Para nosotros y coincidiendo con Schejtman y Berdegué (op cit) a modo de síntesis diríamos que se trata de una construcción social, es decir un lugar construido por todos, caracterizado por un conjunto de relaciones multidimensionales, donde cada una de las partes de dichas relaciones están en interacción y se disputan el poder sobre una porción de ese territorio y en donde existe claramente el sentido de dominación y ,en consecuencia, desigualdades tanto económicas como políticas y socioculturales.

\section{Las políticas públicas y el desarrollo territorial rural}

Es precisamente sobre ese territorio que acabamos de abordar, donde se pretende planificar el desarrollo a partir de las políticas públicas, definida por Tamayo Sáenz (1997) como "el conjunto de objetivos, decisiones y acciones que lleva a cabo un gobierno para solucionar los problemas que en un momento determinado los ciudadanos y el propio gobierno consideran prioritarios"

En general cuando se trata de implementar políticas públicas en el sector rural, lo que se intenta es mejorar las condiciones de vida y aumentar los ingresos de los pequeños productores tratando de reducir los efectos frente a las políticas de ajuste y desregulación y, complementariamente, modernizar, reconvertir y diversificar las explotaciones para al alcanzar niveles de productividad sustentables y con capacidad competitiva. (SAGPyA, 1997).

Por su parte, Peter Hall (1993), propone un enfoque interactivo o institucional, para definir a la Política Pública, situándola en un amplio contexto de relación Sociedad-Estado, "poniendo énfasis en el papel crítico que las Instituciones juegan en la definición y articulación de intereses, en la

Publicado en formato digital: Mgter. Prof. Viviana Pértile. LAS TRANSFORMACIONES TERRITORIALES Y EL DESARROLLO RURAL TERRITORIAL EN LA PROVINCIA DEL CHACO. Revista Geográfica Digital. IGUNNE. Facultad de Humanidades. UNNE. Año 11. No 22. Julio - Diciembre. 2014. ISSN 1668-5180 Resistencia, Chaco. 
propagación de ideas, en la construcción de conductas de mercado y en la determinación de estrategias".

De ello se deriva como factor fundamental para comprender los intercambios entre los diversos actores sociales y estatales, involucrados en las políticas públicas la idea de Instituciones, "en tanto reglas, normas, prácticas informales y tradiciones que resultan de la acción humana" (Repetto, 1998).

Mabel Manzanal (op cit) sostiene que el Desarrollo rural en la Argentina no fue una política de gobierno, sino que fue una sumatoria de programas que hubo desde 1985 hasta 2007, es decir previo a los conflictos del campo y que es cuando se crea la Secretaría de Desarrollo Rural y Agricultura Familiar y que después se transforma en Ministerio de Agricultura (7). Lo primero que se plantea en la década del 80 era por qué Argentina tenía que tener planes de desarrollo rural, desde entonces la respuesta tiene que ver con que eran para mejorar la calidad de vida de los pequeños productores y pobres rurales. Esto es alcanzar mejoras en la producción, en la colocación y articulación en los mercados regionales y nacionales, en la organización a través de la promoción y acceso a recursos productivos, crédito, asistencia técnica y capacitación y de esa manera contribuir al alivio de la pobreza en las áreas rurales.

Es así como comienzan a aparecer los Programas, organizados desde distintos organismos:

- SAGPyA (Secretaría de Agricultura, Ganadería, Pesca y Alimentación) tuvo a su cargo: PSA (Programa Social Agropecuario), PROINDER (Proyecto de Desarrollo de Pequeños Productores Agropecuarios), PRODERNEA (Programa de Desarrollo Rural de las Provincias del Noreste Argentino), estos tres estuvieron orientados a los pequeños productores, CAPPCA (Proyecto Forestal de Desarrollo), PRODERPA, (Proyecto de Desarrollo Rural de la Patagonia) PRODEAR (Programa de Desarrollo de Áreas Rurales). Ministerio de Economía y Producción (2003)

- INTA (Instituto Nacional de Tecnología Agropecuaria) se ocupo de: PROFEDER (Programa Federal de Apoyo al Desarrollo Rural Sustentable, este Programa fue diseñado y opera desde el año 2003 para contribuir a la promoción de la innovación tecnológica y organizacional, el desarrollo de las capacidades de todos los actores del sistema y el fortalecimiento de la competitividad sistémica regional y nacional, en un ámbito de equidad social y sustentabilidad. Su acción se ejecuta mediante proyectos y planes de trabajos grupales en terreno, destinados a satisfacer las demandas y oportunidades que surgen del territorio. La respuesta a la demanda específica se opera mediante distintos instrumentos, con objetivos y metodologías de trabajo propios: Cambio Rural (Programa Federal de Reconversión Productiva para la Pequeña y Mediana Empresa Agropecuaria, si bien se encuentra dentro del PROFEDER, fue creado en el año 1993 por la Secretaría de Agricultura, Ganadería y Pesca), Pro-Huerta (El Pro-Huerta es un programa del INTA y del Ministerio de Desarrollo Social de la Nación con más de 20 años de trabajo en territorio, Basado en los principios de la agricultura orgánica,), Proyectos de Apoyo al Desarrollo Local, Minifundio, Profam (Productores familiares, se inicia en el año 2003 y destinados a productores de muy pequeñas escalas, con dificultades para el acceso al crédito y a la comercialización y con bajos ingresos ) y Proyectos Integrados. Todos y cada uno de los instrumentos basan la estrategia en la acción participativa. INTA (2014)

Todos estos programas estuvieron financiados por el Instituto Nacional de Tecnología Agropecuaria, por la Secretaría de Agricultura, Ganadería, Pesca y Agricultura, por algunos Estados provinciales, Instituciones locales y el Ministerio de Desarrollo Social de la Nación.

EI PSA, funciono en todo el país, con distintas características según sea cada provincia y como era financiado únicamente con presupuesto del Estado no se consideraban deudas, y abarcó a todas las provincias; el PROINDER, estuvo financiado por el Banco Mundial pero tenía más normativas y requisitos para poder acceder a este programa, además promovían la organización, ya que el financiamiento se daba a grupos de cinco o seis productores, dependía de la región, en la

Publicado en formato digital: Mgter. Prof. Viviana Pértile. LAS TRANSFORMACIONES TERRITORIALES Y EL DESARROLLO RURAL TERRITORIAL EN LA PROVINCIA DEL CHACO. Revista Geográfica Digital. IGUNNE. Facultad de Humanidades. UNNE. Año 11. № 22. Julio - Diciembre. 2014. ISSN 1668-5180 Resistencia, Chaco. 
Patagonia eran solamente tres. Como en este caso el financiamiento era extranjero, sí se contraían deudas, lo cual implicaba que las provincias debían asumir dichos compromisos para devolver los préstamos

PRODERNEA y PRODERNOA, PRODERPA, PRODEAR, y CAPPCA son programas, financiados por el Banco interamericano de desarrollo y por FIDA (Fondo Interamericano de Desarrollo Agrario).

Dentro de toda esta serie de Programas, en esta oportunidad sólo hacemos referencia al PRODERNEA, por haber sido el Chaco, uno de los territorios donde se implementó el mismo. Este Programa de Desarrollo Rural de las Provincias del Noreste Argentino, estuvo destinados pobladores pobres rurales que habitaban en áreas del proyecto, que exploten o no la tierra y comunidades aborígenes. Se trató de un Programa nacional y provincial, en donde las provincias tenían que aceptar a través de su legislatura provincial las deudas, el Chaco fue una de ellas. Fue un programa muy importante, visto desde la atención a la cantidad de pequeños productores que había en ese momento. Este programa se inicia en el año 1999 y finaliza en el año 2007 y su propósito fue contribuir a superar las condiciones que generan la pobreza rural, a través del aumento sostenible del ingreso y de la capacidad de autogestión de pobladores rurales e indígenas de las provincias del noreste argentino.

A partir de la implementación de estos programas, cabe preguntarnos si se obtuvieron resultados positivos en la atención a pequeños productores y trabajadores rurales y si existieron debilidades. La posibilidad de tomar contacto directo con muchos de los pequeños productores de la provincia del Chaco, nos ha permitido arribar a algunas conclusiones respecto de lo que este Programa significó para muchos de ellos. En primer lugar debemos señalar que este Programa significó la posibilidad de acceder al crédito y a la asistencia técnica. Asimismo manifestaron algunas problemáticas entre las que se destacaron por ejemplo la imposibilidad de comprar los insumos presupuestados originalmente como consecuencia de la devaluación de nuestra moneda; el aumento del costo del transporte; la falta o inadecuación de la asistencia técnica, en este sentido, ellos consideraban que existía desfasaje entre lo que necesitaban y lo que recibían, tanto por la discontinuidad de la asistencia como por el contenido de las mismas, es decir en algunos casos la producción se orientó de acuerdo con sus conocimientos y no con las necesidades de los productores; otra de las dificultades estaban relacionadas los créditos contraídos, ya que éstos no contribuían a mejorar sus condiciones de vida y de trabajo, de modo que veían más como un elemento de conflicto que cómo un elemento facilitador, encontrándose no solo endeudados, sino también empobrecidos.

En este sentido, Manzanal y Nardi (2008) manifiestan que estos programas contribuyeron a que la cuestión campesina, los pequeños productores pobres y la agricultura familiar se hicieran visibles. Hasta ese momento nadie los veía, estaban invisibilizados. De modo que estos programas sirvieron para demostrar que se necesitan políticas específicas para este sector, distintas a las políticas de Estado más generales que existían para el sector agropecuario. Hasta ese momento se habían formado desde ámbitos académicos a profesionales, ingenieros agrónomos para atender tecnológicamente la problemática de la pampa húmeda, del trigo, de las grandes producciones, dejando de lado temas relacionados con la problemática minifundistas y de los pequeños productores que hasta ese momento no estaban en la enseñanza que se impartía a dichos profesionales. Es a partir de esta situación que comenzaron a formarse y a conocer que existía una realidad que no era únicamente la de la Pampa Húmeda, ni de los grandes cultivos y que dentro de esas producciones se encontraban grupos de productores que poseen otras realidades y otras necesidades y que viven de otra manera, que no viven tanto para el mercado, sino mas para el autoconsumo, donde toda la familia trabaja en forma conjunta (8).

Manzanal y Nardi (Op Cit) destacan claramente las fortalezas y debilidades de estos programas. Entre los principales logros se señalan los siguientes:

- En general otorgaban préstamos únicamente a grupos de pequeños productores, de modo que entre los logros debemos destacar que éstos permitieron generar formas asociativas y actitudes cooperativas.

- Por otra parte se Integró a los beneficiarios directos en la estructura de gestión de los programas, por cuanto además de los funcionarios públicos, fue posible incluir un

Publicado en formato digital: Mgter. Prof. Viviana Pértile. LAS TRANSFORMACIONES TERRITORIALES Y EL DESARROLLO RURAL TERRITORIAL EN LA PROVINCIA DEL CHACO. Revista Geográfica Digital. IGUNNE. Facultad de Humanidades. UNNE. Año 11. № 22. Julio - Diciembre. 2014. ISSN 1668-5180 Resistencia, Chaco. 
representante por región de cada organización de pequeños productores. En ese sentido los programas generaron cierto capital social entre los productores y en algunos ámbitos locales y se avanzó con mayor formalidad. Por ejemplo teniendo la posibilidad de emitir sus propias facturas, a tener jubilación, seguro social, es decir a estar más incorporado al modelo que lo domina.

- Los programas hicieron que las problemáticas del campo y de los campesinos si hicieran visibles.

- Desarrollaron nuevas experiencias innovativas, en este sentido los ingenieros agrónomos fueron los encargados de poner en prácticas dichas experiencias, como lo fue por ejemplo el queso de cabra, la miel orgánica y las ferias francas -lugares donde los productores podían exponer y vender los productos de estas experiencias.

Algunas de las debilidades que pudieron advertirse en los Programas de Desarrollo Rural se encuentran:

- Frente a la cantidad de pobres rurales el Número de beneficiarios fue acotado, esto hace que se los siga invisibilizando.

- Falta de evaluaciones que efectivamente digan lo que se logró, es decir la correspondencia entre lo planeado y lo ejecutado, es decir entre lo que se decía que iba a alcanzar y lo que se consiguió.

- En general existió una falta de conexión entre los programas, algunas veces se superponían, por un lado se encontraba el PSA, por otro el PRODERNEA, se disputaban los beneficiarios, porque no encontraban otros potenciales favorecidos, los que encontraban eran los que más se movilizaban, incluso algunos beneficiarios tenían dos programas, es decir que se advirtió falta de coordinación desde los programas.

- Se observó una sumatoria de ofertas especializadas, es decir escasa complementación entre los Programas y dificultades para lograrla.

- Otras de las importantes falencias que estos programas tuvieron fue la discontinuidad en el financiamiento, de modo que funcionaban con total incertidumbre, afectando al normal desenvolvimiento de los proyectos. El financiamiento provenía del Banco Mundial y del Gobierno Nacional. Sabemos además que nuestro gobierno ha pasado por sucesivas crisis, inestabilidad económica, social y política, de modo que no se tenía la certeza de que al año siguiente se podía contar con los fondos necesarios para continuar el programa. De esa manera se hacía difícil consolidar una política donde existiese una incertidumbre permanente, básicamente porque se trataba de una actividad de capitalización, formando un capital social para la gente.

- Otras de las falencias fue la escasa difusión de resultados y estudios, es decir no se conocía cómo se trabajaba o cómo se evaluaba.

A modo de cierre entre estas fortalezas y debilidades de algunos de estos programas de desarrollo rural, en el año 2006 se realizó en la ciudad de Posadas, Misiones el Primer Encuentro Regional de PRODERNEA (9) cuyo objetivo fue generar un espacio de reflexión entre los beneficiarios, como así también la oportunidad de destacar aspectos sobresalientes de su implementación y algunas experiencias productivas. En ese marco también se expusieron las dificultades que habían tenido con este programa, muchas de las cuales coincidían con las mencionadas anteriormente, entre las que se destacaban:

- Retrasos en los desembolsos de los créditos.

- Dificultades para consolidar los grupos

- Aspectos relacionados a comercialización y mercados

- Asistencia técnica con pocas reuniones e inadecuado seguimiento

- Poca difusión del Programa 
Revista Geográfica Digital. IGUNNE. Facultad de Humanidades. UNNE. Año 11. № 22.

Julio - Diciembre 2014. ISSN 1668-5180 Resistencia, Chaco

\section{Conclusión}

En todo proceso de transformación existen continuas modificaciones y ajustes como consecuencia de que las acciones que buscan alcanzar ciertos objetivos, se confrontan generalmente con intereses y demandas de numerosos actores sociales.

Es indiscutible la demostración de poder que ejercen ciertos sectores privilegiados al señalar qué debemos hacer para desarrollarnos. Esto queda en evidencia cuando se pone la mirada en el territorio como centro de acción para llevar a cabo políticas de desarrollo, ya sea porque somos un país subdesarrollado o por las diferencias regionales existentes.

Es importante entender que la permanente propuesta de alternativas para lograr el desarrollo rural se ha convertido es un instrumento del poder dominante para plantear ilusiones que mantengan a la población rural de menores recursos imaginando un futuro más ventajosos y superador. A través de los años hemos observado que los diferentes gobiernos han garantizado la rentabilidad capitalista asegurando un proceso expansión acelerada de superficies cultivadas y de acumulación y al mismo tiempo una aceptación general de una desigualdad socio-territorial cada vez más marcada, sobre todo de sectores rurales marginales y en particular de la provincia del Chaco, prueba de ello lo constituye el número de población rural cada vez menor habitando el territorio regional y provincial.

En base a la bibliografía y documentación analizada, podemos concluir que tanto los gobiernos de turno como los sectores económicos de poder consideran al desarrollo como sinónimo de crecimiento económico en contraposición a la desigualdad social que dicho proceso genera y sin considerar que solamente un cambio radical y estructural puede lograrlo.

\section{Referencias}

1. Resulta ser la capacidad que ostenta un país, una comunidad, para mejorar el bienestar social de su pueblo, por ejemplo y entre otras cuestiones, ofreciéndoles excelente condiciones laborales, oportunidades de empleo concretas, acceso a la educación, a una vivienda digna, a la salud y la distribución equitativa de la riqueza nacional. En: http://www.definicionabc.com/general/desarrollonacional.php.

2. CFI: orientado a la sustentabilidad de los procesos de desarrollo regional

3. CONADE: organismo esencialmente consultivo y técnico, que tenía la misión de precisar los objetivos a largo plazo del desarrollo y analizar las condiciones en que deberían desenvolverse todos los sectores sociales para lograrlo.

4. Esto fue así porque este programa de ayuda económica y social por 10 años (1961-1970) para América latina estuvo dirigido a enfrentar los riesgos que significaba la reciente revolución cubana (1959) para EEUU y para todo el sistema capitalista. (Manzanal y Schneider, Op cit).

5. En el año 2012 la CEPAL difundió un documento donde manifiesta claramente las desigualdades existentes en América Latina y dice: "Uno de los grandes desafíos que continúa enfrentando América Latina es la reducción de los elevados niveles de desigualdad en la distribución del ingreso prevalecientes en la región. En la mayoría de los países se observa que un conjunto reducido de la población acumula una gran proporción de todos los ingresos generados, mientras que los más pobres sólo alcanzan a recibir una escasa porción. El promedio simple de los valores de los 18 países de los que se cuenta con información relativamente reciente indica que el $10 \%$ más rico de la población recibe el $32 \%$ de los ingresos totales, mientras que el $40 \%$ más pobre recibe el $15 \% "$. (CEPAL, 2012).

6. Para el PROINDER 2007: Pequeño productor familiar de subsistencia, es aquel cuya dotación de recursos no le permite vivir exclusivamente de su explotación y mantenerse en la actividad, debe recurrir a otras estrategias de supervivencia, posee acentuadas condiciones de pobreza, y su mantenimiento en el campo se explica por el aporte que recibe de programas públicos de asistencia social y por otros ingresos eventuales. Pequeño productor familiar descapitalizado, posee una escasez de recursos tal que no le permite una reproducción ampliada o la evolución de su explotación 
sino solamente la explotación simple, es decir mantener la actividad, y presenta algunos rasgos de pobreza por falta de acceso a servicios sociales básicos

7. El Ministerio de Agricultura, Ganadería y Pesca (MAGyP) de la Argentina es el organismo gubernamental responsable de diseñar y ejecutar planes de producción, comercialización y sanidad en el ámbito agropecuario, pesquero, forestal y agroindustrial.

Hasta 2008, era una Secretaría dependiente del Ministerio de Economía. A partir del 1 de octubre de 2009, fue elevado al rango de Ministerio. Este Ministerio cuenta con la Secretaría de Desarrollo Rural y Agricultura Familiar, de la cual depende la Subsecretaría de Agricultura Familiar y la Subsecretaría de Desarrollo de Economías Regionales.

http://www.minagri.gob.ar/site/institucional/index.php

8 El autoconsumo no solo implica el consumo en el ámbito familiar de lo producido, sino también la venta en las ciudades vecinas de los productos obtenidos en sus pequeñas producciones.

9 Ministerio de Agricultura, Ganadería y Pesca http://64.76.123.202/new/0-

0/programas/prodernea/publicaciones/publicaciones.php?carpeta=Desarrollo_Rural

\section{Bibliografía}

Baigorri, a. (1995): De lo rural a lo urbano, hipótesis sobre las dificultades de mantener la separación epistemológica entre Sociología Rural y Sociología Urbana en el marco del actual Proceso de Urbanización Global. In: V Congreso Español de Sociología. Granada, 1995.

Bozzano, Horacio (2009): Territorios posibles. Procesos, lugares y actores. Ed. Lumiere. Buenos Aires. Argentina.

Caravaca Barroso, Inmaculada. Los nuevos espacios ganadores y emergentes. EURE (Santiago) [online]. 1998, vol.24, n.73 [citado 2014-04-04], $p$ pp. $<$ http://www.scielo.cl/scielo.php?script=sci_arttext\&pid=S0250-

71611998007300001\&lng=es\&nrm=iso>. $\quad$ ISSN 0250-7161. http://dx.doi.org/10.4067/S025071611998007300001.

CASTELLS, Manuel (1998). "Entender nuestro mundo", en Revista de Occidente, España. Fundación José Ortega y Gasset.

CEPAL (2012): Panorama Social de América Latina. Documento informativo.

http://www.eclac.org/publicaciones/xml/5/48455/PanoramaSocial2012Docl-Rev.pdf

Clout, Hugh D (1976).: Geografía Rural. 5a ed. Oikos-Tau. Barcelona.

Diry, Jean Paul "Los espacios rurales". Ed. SEDES. París 1999.

Dubois, Alfonso (2006): Un Concepto de Desarrollo Para el Siglo XXI. En: Revista Asuntos: Revista Asuntos Económicos y Administrativos. Universidad de Manizales. Facultad de Economía y Administración de Empresas.

Esteva, Gustavo, 1996. Desarrollo. En SACHS, W. (editor) Diccionario del desarrollo. Una guía del conocimiento como poder. Perú: PRATEC, 1996. 383p. p. 52-79. Blanco, Jorge (2007). "Espacio y territorio: elementos teórico conceptuales implicados en el análisis geográfico". En María Victoria Fernández y Raquel Gurevich (coords.), Geografía: nuevos temas, nuevas preguntas. Un temario para su enseñanza (pp. 37-64). Buenos Aires: Editorial Biblos.

Fernandes, Bernardo Mançano (2010). Territorios en disputa: campesinos y agrobusiness. en : http://www.landaction.org/IMG/pdf/Bernardo halifax esp.pdf.

González De Canales López-Obrero, F. (2003). Concepto de territorio. Nota técnica del Instituto de Desarrollo Comunitario. Fundación INFODAL. Madrid. En: Martínez de Anguita, Pablo (2008): Principios generales para un Desarrollo Rural. http://fundacionapic.blogspot.com.ar/2008/06/principiogenerales-para-un-desarrollo.html

Hall, Peter. (1993). El gobierno de la economía. Implicaciones políticas de la intervención estatal en la economía de Gran Bretaña y Francia, Madrid.

INTA 2014: Programa Federal de Apoyo al Desarrollo Rural http://inta.gob.ar/documentos/profederprograma-federal

Publicado en formato digital: Mgter. Prof. Viviana Pértile. LAS TRANSFORMACIONES TERRITORIALES Y EL DESARROLLO RURAL TERRITORIAL EN LA PROVINCIA DEL CHACO. Revista Geográfica Digital. IGUNNE. Facultad de Humanidades. UNNE. Año 11. № 22. Julio - Diciembre. 2014. ISSN 1668-5180 Resistencia, Chaco. 
Keynes,John Maynard, 2005: Teoría general de la ocupación, el interés y el dinero. Ed. Fondo de Cultura Económica. Bs. As. Argentina

Laguna Marín-Yaseli, María (2007): Más de 20 años de políticas de desarrollo rural: la experiencia de las políticas regionales en el Pirineo aragonés. Editorial: Universidad de Zaragoza. España

Manzanal y Sneider (2010) Agricultura Familiar y Políticas de Desarrollo Rural en Argentina y Brasil (análisis comparativo, 1990-2010).

Manzanal y Sneider (2011) Agricultura Familiar y Políticas de Desarrollo Rural en Argentina y Brasil (análisis comparativo, 1990-2010). Revista Interdisciplinaria de Estudios Agrarios $N^{\circ} 34$, p. 35-71, CIEA, FCE, UBA, ISSN 1514-1535, 1er semestre 2011, Buenos Aires.

Manzanal, Mabel (2011) Desarrollo, territorio y desigualdad en la globalización. Conflictos actuales en la agricultura familiar del nordeste de Misiones, Argentina. En: Revista Mundo Agrario, vol. 12, $\mathrm{n}^{\circ} 23$, segundo semestre de 2011. Universidad Nacional de La Plata. Facultad de Humanidades y Ciencias de la Educación. Centro de Historia Argentina y Americana.

Manzanal, Mabel (2013). "Poder y desarrollo. Dilemas y desafíos frente a un futuro ¿cada vez más desigual?". En Manzanal, M. y Ponce, M. (2013) La desigualdad ¿del desarrollo?. Controversias y disyuntivas del desarrollo rural en el norte argentino. Ediciones CICCUS, Buenos Aires.

Manzanal, Mabel y Nardi, María (2008): Modelos de intervención en el desarrollo rural. En: El desarrollo rural en la Argentina, un enfoque territorial. Schejtman, Alejandro y Barsky, Osvaldo (compiladores): Ed. Siglo XXI. Buenos Arres.

Manzanal, Mabel y Otros (2011): Poder y conflicto en territorios del Norte Argentino. E: Estudios Socioterritoriales. Revista de Geografía. № 9. ene-jun 2011, pág. 57-81. Universidad Nacional del Centro de la Provincia de Buenos Aires. Facultad de Ciencias Humanas. Centro de Investigaciones Geográficas -CIG.

Manzanal, Mabel y Ponce, Mariana (2013): La desigualdad ¿del desarrollo?. Controversias y disyuntivas del desarrollo rural en el Norte Argentino. Ediciones CICCUS, Buenos Aires

Maryvonne Le Berre, (1992). Concepto de territorio. En Hypergeo, Enciclopedia electrónica http://www.hypergeo.eu/spip.php?article514

Ministerio de Agricultura, Ganadería y Pesca: Primer Encuentro Regional de PRODERNEA En: http://64.76.123.202/new/00/programas/prodernea/publicaciones/publicaciones. php?carpeta=Desarrol lo_Rural

Ministerio De Economía Y Producción (2003): Los programas de desarrollo rural ejecutados en el ámbito de la SAGPYA. Serie estudios e investigaciones. Buenos Aires

Neira Alva, Eduardo (1974). "Las políticas de desarrollo regional en América Latina", Planificación regional y urbana en América latina, ILPES-ILDES, Siglo XXI, México. Citado en

Pertile, V. y Torre Geraldi, A (2009): "Cambios productivos en el sector agrícola de la provincia del Chaco: la sojización y sus consecuencias socioeconómicas en los pequeños productores rurales a fines del siglo XX". En: Morello, Jorge y Andrea F. Rodríguez (editores) El Chaco sin Bosques: La Pampa o el Desierto del futuro GEPAMA- Universidad de Buenos Aires -UNESCO. Editorial Orientación Grafica

Proinder /Dirección De Desarrollo Agropecuario (2007): Los pequeños productores en la República Argentina

Repetto, Fabián. (1998). Notas para el análisis de la política social: Una propuesta desde el institucionalismo. EN Perfiles Latinoamericanos. FLACSO. Año 7 № 12

Sancho Comíns, j., Martínez, J. y Martín, M. A. (2002). Necesidad de un Marco Jurídico para el Desarrollo Rural en España. Consejo Superior de Investigaciones Científicas. Madrid. En : Martínez de Anguita, Pablo (2008): Principios generales para un Desarrollo Rural. http://fundacionapic.blogspot.com.ar/2008/06/principio-generales-para-un-desarrollo.html Santos, Milton (1986). Por uma geografia nova. Da crítica da Geografia a uma Geografia Crítica. Terceira edicao. ED. HUCITEC, São Paulo, Brasil.

Schejtman, Alexander y BERDEGUÉ, Julio (2004). Desarrollo territorial rural. Debates y temas rurales No 1. Rimisp / Centro Latinoamericano para el desarrollo rural, Santiago de Chile. Disponible en http://www.rimisp.org

Scherjtman, Alejandro y Barsky, Osvaldo (2008): El desarrollo rural en la Argentina. Ed. Siglo XXI. Buenos Aires, Argentina 
Secretaría de Agricultura, Ganadería y Pesca, (1997): Estrategia de Desarrollo Rural Hacia el Siglo XXI. SAGPyA. Buenos Aires.

Sosa Velasquez, Mario (2012): ¿Cómo entender el territorio? Ed. Cara Parens. Universidad Rafael Landívar. Guatemala.

Tamayo Saenz, Manuel (1997).El análisis de las políticas Públicas. En: Bañón, Rafael y Carrillo, Ernesto (compiladores). La Nueva Administración Pública, Alianza Universidad, Madrid 